\title{
Establishment of a Monitoring Clinic Based on Wearables for Patients with Heart Failure
}

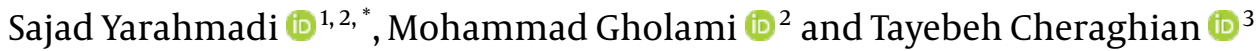 \\ ${ }^{1}$ Social Determinants of Health Research Center, Lorestan University of Medical Sciences, Khorramabad, Iran \\ ${ }^{2}$ School of Nursing and Midwifery, Lorestan University of Medical Sciences, Khorramabad, Iran \\ ${ }^{3}$ Shahid Rahimi Hospital, Lorestan University of Medical Sciences, Khorramabad, Iran \\ "Corresponding author: School of Nursing and Midwifery, Lorestan University of Medical Sciences, Khorramabad, Iran. Email: s.yarahmadiooo@gmail.com
}

Received 2020 September 20; Accepted 2020 October 29.

Keywords: Biosensors, Cardiac Clinic, Heart Failure, Wearable Devices

\section{Dear Editor,}

Heart failure affects approximately $1 \%-2 \%$ of the population of developed countries (1). The number of patients with heart failure in Iran is reported to be 3,500 per 100,000 population, ranging from $2 \%$ to $8 \%$. About $30 \%$ of patients refer to clinics each year, and about $40 \%$ of these patients are hospitalized with dysfunction of body organs (2). These statistics show that the current management of patients with heart failure is not effective, and even shortterm and momentary visits of patients in clinics cannot reflect the patient's condition at all times.

Progress in technology has provided solutions to these problems. The use of implants in patients with heart failure to titrate various drugs based on direct arterial blood pressure is an example of these technologies (3). These devices are currently very expensive, and due to their aggressive state, they cannot be used by everyone. Using less invasive devices and increasing the ability to control patients with heart failure remotely can be very helpful. In the United States, about $20 \%$ of people now use fitness monitors to track their health information. There is other equipment that, by adding biological sensors and the ability to send signals to the clinic, can better assess the condition of heart failure patients and frequently consider appropriate treatment and care. Examples of available devices that can be used by cardiovascular patients include bracelets that are speedometers and can measure the heart rate; smartwatches for measuring heart rate, ECG, and blood pressure; electronic patches that measure ECG and lung fluid, suitable Wearable devices for ECG examination and measurement of pulmonary fluids (4).

1. Activity Level Measurement: The use of a step counter is increasing. The device has an accelerometer (Fitbit ${ }^{\mathrm{TM}}$ ) and measures the amount of movement and converts it to the amount of physical activity according to an algorithm. There are many commercial devices in this vein. Exercise and activity can reduce the risk of hospitalization in patients with heart failure (5), and the use of wearable devices to monitor physical activity by registering and encouraging patients to be more active can facilitate this goal (Figure1). One study found that people who used these devices increased the number of steps per day from 5,899 to 7,890 (6). Another study showed that 60 minutes of physical activity per day in patients with congestive heart failure reduced the risk of mortality by $35 \%$ (7).

2. Heart Rate Monitoring: These devices are usually attached to the wrist and control heart rate using photolithography (PPG). Using a light-emitting diode, the device illuminates a capillary substrate and thus detects ECG changes (such as pulse oximeters). In a study that compared the accuracy of these devices, four models of these devices were compared with ECGs. The results showed that with a difference of $20 \%$ per minute, these devices were similar to ECGs (8).

3. Blood Pressure Monitoring: High blood pressure is one of the causes of heart failure. Heart failure and its treatments are reasons for hypotension (4). Blood pressure measurements may be done at home or the clinic once a day, but the use of blood pressure monitor watches (Omron ${ }^{\mathrm{TM}}$ HeartGuide) can do this several times a day and monitor the patient continuously.

4. Diagnosis of Arrhythmias: One of the most common cardiac arrhythmias in patients with congestive heart failure is atrial fibrillation (9). Following the diagnosis of this arrhythmia, the use of anticoagulants is strongly recommended (10). Deformation in PPG waves can indi-

Copyright (C) 2020, Journal of Archives in Military Medicine. This is an open-access article distributed under the terms of the Creative Commons Attribution-NonCommercial 4.0 International License (http://creativecommons.org/licenses/by-nc/4.0/) which permits copy and redistribute the material just in noncommercial usages, provided the original work is properly cited. 


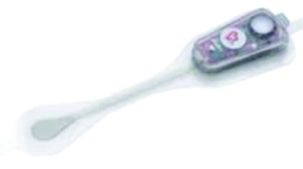

Carnation patch ${ }^{\mathrm{T}}$ : A lightweight heart monitor that focuses more on the P-wave and helps diagnose arrhythmias.

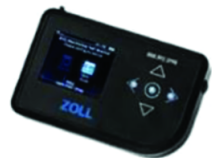

Zoll $\mu \mathrm{Cor}^{\mathrm{TM}}$ : This device continuously records, stores and transmits patient data such as heart rate, chest fluid index, respiration rate, heart rate (ECG), activity and status.

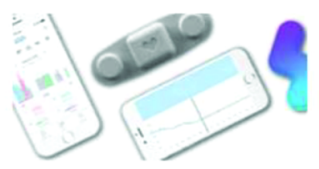

VivaLNK $^{\mathrm{TM}}$ : A 75-gram device that can examine the ECG and can be used for up to 72 hours with a battery.

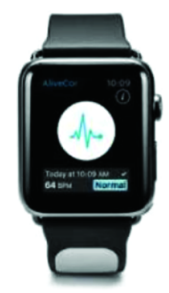

Verily ${ }^{\mathrm{TM}}$ Study watch: ECG and physical activity watch

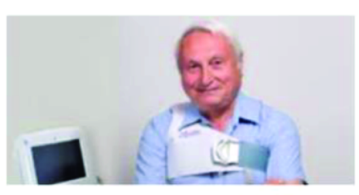

ReDS ${ }^{\mathrm{TM}}$ : A device for checking the volume of non-invasive lung fluid

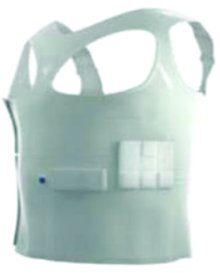

Cardioskin $^{\mathrm{TM}}$ : Wearable ECG device for diagnosis of syncope, float and atrial fibrillation, ischemic heart disease, cardiomyopathy

Figure 1. Wearables in heart failure patients

cate an irregular pulse. Traditional patient monitoring requires a HOLTER to diagnose arrhythmias, but recently FDA-approved wearable chips (Carnation patch ${ }^{\mathrm{TM}}$ and VivaLNK) can be used to monitor patients for several days, making it easier to use these devices. They can be used for a longer time than HOLTER. Some smartwatches (Apple $\mathrm{e}^{\mathrm{TM}}$ watch and the Verily ${ }^{\mathrm{TM}}$ Study watch) have electrodes that can record ECG. One electrode is placed under the watch to be in contact with the skin, and the other electrode is in the crown part of the watch. When the person touches the crown with the other hand, several ECG leads are generated.

5. Monitoring Response to Treatment: Checking the heart rate and blood pressure following the use of various drugs can help patients in drug titration. Monitoring activity levels can be a tool for assessing response to treatment. One study found that patients with heart failure who consumed isosorbide had lower levels of activity (11).

6. Evaluation of Symptoms and Prognosis of the Disease: Assessing the activity capacity of patients with heart failure is inversely related to the stage of the disease so that patients with the upper class of the disease are less able to perform an activity (12). The classification of the heart failure class is subjective, and there is a big difference between the diagnoses of different clinicians. Thus, using these devices to more objectively assess the level of activity and help diagnose the degree of the disease can be helpful.

7. Early Detection And prevention of Irreparable Damage: Hospitalization is costly for patients and the treatment system, and preventing readmission can greatly reduce the costs. Monitoring pulmonary artery pressure and heart rate through implants in the thoracic cavity is very helpful in identifying the onset of risk. The use of wearable devices is a non-invasive alternative to these devices that can quickly detect pulmonary congestion by examining the impedance inside the chest. Intrathoracic impedance correlates well with body fluid levels (13). One study showed the use of these devices with a sensitivity of $87 \%$ and a specificity of $70 \%$ on 106 patients diagnosed with heart failure (14). In a study, to prevent errors in diagnosis, devices were performed to check several parameters such as ECG and intrathoracic impedance and perform accelerometry on 100 patients, which showed 70\% specificity and $87 \%$ sensitivity in 10 days before onset, which is actu- 
ally a high sensitivity for that time (15). An FDA-approved patch (ZOLL $\mu$ Cor $^{\mathrm{TM}}$ ) is used for the early detection of heart failure using radiofrequency waves and ECG monitoring.

Devices for measuring blood pressure, ECG, heart rate, and arterial oxygen saturation are currently available on the market for consumers. These devices must be safe and secure, and the output information must be valid for health workers. Providing devices with low error rates and valid information is usually expensive and may not be usable for most patients. Also, the information obtained from the equipment needs to be interpreted so that the results can change the patient care and treatment program at any time. Therefore, establishing clinics that have the above-mentioned equipment in such a way that this equipment can send information can be a great help to patients with heart failure. Remote monitoring of patients, in addition to reducing hospitalization costs and face-to-face visits to clinics, can also enhance the quality of life and provide comfort for patients with congestive heart failure. Also, due to the COVID-19 disease epidemic, patients may be less inclined to be physically present in medical centers, which can be a risk factor for these patients. Therefore, the establishment of a remote monitoring clinic for patients with congestive heart failure can reduce these problems. Besides, instead of purchasing the devices by patients, these devices should be purchased for a clinic and be provided to patients based on their needs. In addition to reducing the financial burden on patients and the treatment system, jobs can be created for medical graduates.

\section{Footnotes}

Authors' Contribution: Study concept and design: MG. Acquisition of data: SY. Drafting of the manuscript: SY and TC.

Conflict of Interests: The authors of this manuscript declare that there is no conflict of interest.

Funding/Support: None declared by the author.

\section{References}

1. Mosterd A, Hoes AW. Clinical epidemiology of heart failure. Heart. 2007;93(9):1137-46. doi: 10.1136/hrt.2003.025270. [PubMed: 17699180]. [PubMed Central: PMC1955040].

2. Maggioni AP, Dahlstrom U, Filippatos G, Chioncel O, Crespo Leiro M, Drozdz J, et al. EURObservational Research Programme: regional differences and 1-year follow-up results of the Heart Failure Pilot Survey (ESC-HF Pilot). Eur J Heart Fail. 2013;15(7):808-17. doi: 10.1093/eurjhf/hft050. [PubMed: 23537547].
3. Abraham WT, Stevenson LW, Bourge RC, Lindenfeld JA, Bauman JG, Adamson PB, et al. Sustained efficacy of pulmonary artery pressure to guide adjustment of chronic heart failure therapy: complete follow-up results from the CHAMPION randomised trial. Lancet. 2016;387(10017):453-61. doi: 10.1016/S0140-6736(15)00723-0. [PubMed: 26560249].

4. Singhal A, Cowie MR. The Role of Wearables in Heart Failure. Curr Heart Fail Rep. 2020;17(4):125-32. doi: 10.1007/s11897-020-00467-x. [PubMed: 32494944]. [PubMed Central: PMC7343723].

5. Taylor RS, Sagar VA, Davies EJ, Briscoe S, Coats AJ, Dalal H, et al. Exercisebased rehabilitation for heart failure. Cochrane Database Syst Rev. 2014;(4). CD003331. doi: 10.1002/14651858.CD003331.pub4. [PubMed: 24771460]. [PubMed Central: PMC6485909].

6. Thorup C, Hansen J, Gronkjaer M, Andreasen JJ, Nielsen G, Sorensen EE, et al. Cardiac Patients' Walking Activity Determined by a Step Counter in Cardiac Telerehabilitation: Data From the Intervention Arm of a Randomized Controlled Trial. J Med Internet Res. 2016;18(4). e69. doi: 10.2196/jmir.5191. [PubMed: 27044310]. [PubMed Central: PMC4835668].

7. Loprinzi PD. The effects of free-living physical activity on mortality after congestive heart failure diagnosis. Int J Cardiol. 2016;203:598-9. doi: 10.1016/j.ijcard.2015.11.017. [PubMed: 26574935].

8. Cadmus-Bertram L, Gangnon R, Wirkus EJ, Thraen-Borowski KM, Gorzelitz-Liebhauser J. Accuracy of Heart Rate Monitoring by Some Wrist-Worn Activity Trackers. Ann Intern Med. 2017;167(8):607-8. doi: 10.7326/L17-0380. [PubMed: 29049770].

9. Anter E, Jessup M, Callans DJ. Atrial fibrillation and heart failure: treatment considerations for a dual epidemic. Circulation. 2009;119(18):2516-25. doi: 10.1161/CIRCULATIONAHA.108.821306. [PubMed: 19433768].

10. Ponikowski P, Voors AA, Anker SD, Bueno H, Cleland JGF, Coats AJS, et al. 2016 ESC Guidelines for the diagnosis and treatment of acute and chronic heart failure: The Task Force for the diagnosis and treatment of acute and chronic heart failure of the European Society of Cardiology (ESC)Developed with the special contribution of the Heart Failure Association (HFA) of the ESC. Eur HeartJ. 2016;37(27):2129-200. doi: 10.1093/eurheartj/ehw128. [PubMed: 27206819].

11. Redfield MM, Anstrom KJ, Levine JA, Koepp GA, Borlaug BA, Chen $\mathrm{HH}$, et al. Isosorbide Mononitrate in Heart Failure with Preserved Ejection Fraction. $N$ Engl J Med. 2015;373(24):2314-24. doi: 10.1056/NEJMoa1510774. [PubMed: 26549714]. [PubMed Central: PMC4712067].

12. Baril JF, Bromberg S, Moayedi Y, Taati B, Manlhiot C, Ross HJ, et al. Use of Free-Living Step Count Monitoring for Heart Failure Functional Classification: Validation Study. JMIR Cardio. 2019;3(1). e12122. doi: 10.2196/12122. [PubMed: 31758777]. [PubMed Central: PMC6834224].

13. Gastelurrutia P, Cuba-Gyllensten I, Lupon J, Zamora E, Llibre C, Caballero A, et al. Wearable vest for pulmonary congestion tracking and prognosis in heart failure: A pilot study. Int J Cardiol. 2016;215:77-9. doi: 10.1016/j.ijcard.2016.04.024. [PubMed: 27111163].

14. Darling CE, Dovancescu S, Saczynski JS, Riistama J, Sert Kuniyoshi F, Rock J, et al. Bioimpedance-Based Heart Failure Deterioration Prediction Using a Prototype Fluid Accumulation Vest-Mobile Phone Dyad: An Observational Study. JMIR Cardio. 2017;1(1). e1. doi: 10.2196/cardio.6057. [PubMed: 31758769]. [PubMed Central: PMC6832026].

15. Stehlik J, Schmalfuss C, Bozkurt B, Nativi-Nicolau J, Wohlfahrt P, Wegerich S, et al. Continuous Wearable Monitoring Analytics Predict Heart Failure Hospitalization: The LINK-HF Multicenter Study. Circ Heart Fail. 2020;13(3). e006513. doi: 10.1161/CIRCHEARTFAILURE.119.006513. [PubMed:32093506]. 